\title{
Free Vibration Analysis of Anisotropic Right Triangular Plates
}

\author{
異方性直角三角形板の自由振動問題の一解析法
}

\author{
Mei Huang ${ }^{* 1}$, Takeshi Sakiyama ${ }^{* 2}$, Hiroshi Matsuda ${ }^{* 3}$, Chihiro Morita ${ }^{* 4}$ \\ 黄 美, 崎山 毅, 松田 浩, 森田 千尋
}

${ }^{* 1}$ Member of JSCE, Dr.of Eng., JSPS Fellow, Dept.of Struct. Eng. Nagasaki Univ. $(852-8521$, Nagasaki)
${ }^{* 2}$ Member of JSCE, Dr.of Eng., Prof., Dept.of Struct. Eng. Nagasaki Univ. $(852-8521$, Nagasaki)
${ }^{* 3}$ Member of JSCE, Dr.of Eng., Assoc.Prof., Dept.of Struct. Eng. Nagasaki Univ. $(852-8521$, Nagasaki)
${ }^{* 4}$ Member of JSCE, Dr.of Eng., Assoc.Prof., Dept.of Struct. Eng. Nagasaki Univ. (852-8521, Nagasaki)

An approximate method is used for analyzing the free vibration problem of isotropic and anisotropic right triangular plates with various aspect-ratios and variable thickness. In this paper, a right triangular plate is considered as a kind of equivalent rectangular plate with point supports. Therefore, the free vibration characteristics of any triangular plate can be obtained by analyzing the equivalent rectangular plate. The differential equations of the equivalent rectangular plate with point supports are established based on the first-order shear deformation theory. By transforming the differential equations into integral equations and using numerical integration, the solutions of the partial differential equation can be obtained in discrete form. The Green function, which is the discrete solution for the deflection, is introduced to obtain the characteristic equation of the free vibration. Frequencies and mode shapes are shown for some triangular plates with various aspect-ratio and variable thickness. The effects of boundary conditions and the fibre orientation on the frequency parameters are considered. The efficiency and accuracy of the numerical solutions by the present method are investigated.

Key Words : approximate method, triangular plate, point support, equivalent rectangular plate, Green function, vibration

\section{Introduction}

The free vibration problems of isotropic triangular plates have been studied for many years. Early st.udies were well compiled in Ref. 1). Further stud ies have been done for the past two decades. Gorman ${ }^{2), 3), 4)}$ and Saliba ${ }^{5), 6)}$ analyzed the vibration of isotropic right triangular plates with combinations of clamped and simply supported boundary conditions. The superposition method and the modified superposition method were used respectively. Kim and Dickinson ${ }^{7)}$ presented a comprehensive analysis of free vibration of isotropic triangular plates with any combination of free, simply supported or clamped boundary conditions. The free vibration of isotropic triangular plates with linearly varying thickness were also studied by Mirza and Bijlani ${ }^{8)}$, Liew, Lim and Lim 9) and McGee and Giaimo ${ }^{10)}$.
With the increasing use of anisotropic materials in engineering, the study of free vibration problems of anisotropic triangular plates has received more and more attention. The free vibration of orthotropic cantilever triangular plate was analyzed by Kim and Dickinson 7) as one example. The Rayleigh-Ritz method with simple polynomials was used and the lowest six natural frequencies were obtained for the plates with various aspect ratios. Lam, Liew and Chow ${ }^{11)}$ presented the natural frequencies and mode shapes for the orthotropic right triangular plates with three kinds of boundary conditions. A set of twodimensional orthogonal was used as an admissible displacement function in the Rayleigh-Ritz method. The vibration of anisotropic isosceles triangular plates was analyzed by Malhotra, Ganesan and Veluswami 12) by using the finite element method. Bambill, Laura and Rossi ${ }^{13)}$ considered the vibration of orthotropic 
cantilever isosceles triangular plates using the optimized Rayleigh-Ritz method and trigonometric coordinate functions. The first frequency parameter was obtained. But compared with the study of the vibration problems of isotropic triangular plates, the study of the anisotropic plates is rather limited.

In this paper, an approximate method is used for analyzing the free vibration of isotropic and anisotropic right triangular plates with various boundary conditions, aspect-ratios and variable thickness. By adding an additional triangular part, a right triangular plate can be considered finally as a kind of rectangular plate with non-uniform thickness and point supports. The thickness of the additional part is extremely thinner than that of the original part. The boundary conditions along the original hypotenuse are enforced by the appropriate use of some intermediate point supports. Therefore, the free vibration characteristics of any right triangular plate are obtained by analyzing an equivalent rectangular plate with non-uniform thickness. The characteristic equation of the free vibration is obtained by using Green function, which is the discrete-form solution ${ }^{14)}$ for the deflection of the plate with a concentrated load at each discrete point. The frequencies and their mode shapes are shown for some triangular plates with various aspect-ratio and variable thickness. The efficiency and accuracy of the numerical solutions by the present method are investigated.

\section{Discrete Green Function}

The coordinate system for an anisotropic plate used in the present study is shown in Fig. 1. The $x y z$ coordinate system is assumed to have its origin at the corner of the middle plane of the plate. The surfaces of the plate are at $z= \pm h / 2$ and $h$ is the thickness of the plate. 1-, 2- and 3-directions are principal axes in the longitudinal, transverse and normal directions, respectively. The differential equations of the plate with a concentrated load $\bar{P}$ at point $\left(x_{q}, y_{r}\right)$ and point support at each discrete point $\left(x_{c}, y_{d}\right)$ are:

$$
\begin{aligned}
\frac{\partial Q_{x}}{\partial x} & +\frac{\partial Q_{y}}{\partial y}+\bar{P} \delta\left(x-x_{q}\right) \delta\left(y-y_{r}\right) \\
& +\sum_{c=0}^{m} \sum_{d=0}^{n} \bar{P}_{1 c d} \delta\left(x-x_{c}\right) \delta\left(y-y_{d}\right)=0 \\
\frac{\partial M_{x}}{\partial x} & +\frac{\partial M_{x y}}{\partial y}-Q_{x} \\
& +\sum_{c=0}^{m} \sum_{d=0}^{n} \bar{P}_{2 c d} \delta\left(x-x_{c}\right) \delta\left(y-y_{d}\right)=0
\end{aligned}
$$

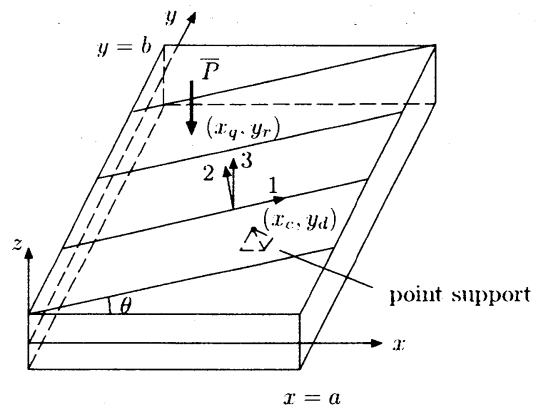

Fig. 1 Coordinate system for an anisotropic rectangular plate

$$
\begin{aligned}
\frac{\partial M_{y}}{\partial y}+ & \frac{\partial M_{x y}}{\partial x}-Q_{y} \\
& -\sum_{c=0}^{m} \sum_{d=0}^{n} \bar{P}_{3 c d} \delta\left(x-x_{c}\right) \delta\left(y-y_{d}\right)=0 \\
M_{x}= & D_{11} \frac{\partial \theta_{x}}{\partial x}+D_{12} \frac{\partial \theta_{y}}{\partial y}+D_{16}\left(\frac{\partial \theta_{x}}{\partial y}+\frac{\partial \theta_{y}}{\partial x}\right)(4) \\
M_{y}= & D_{12} \frac{\partial \theta_{x}}{\partial x}+D_{22} \frac{\partial \theta_{y}}{\partial y}+D_{26}\left(\frac{\partial \theta_{x}}{\partial y}+\frac{\partial \theta_{y}}{\partial x}\right)(5) \\
M_{x y}= & D_{16} \frac{\partial \theta_{x}}{\partial x}+D_{26} \frac{\partial \theta_{y}}{\partial y}+D_{66}\left(\frac{\partial \theta_{x}}{\partial y}+\frac{\partial \theta_{y}}{\partial x}\right)(6) \\
Q_{y}= & k A_{44}\left(\frac{\partial w}{\partial y}+\theta_{y}\right)+k A_{45}\left(\frac{\partial w}{\partial x}+\theta_{x}\right) \\
Q_{x}= & k A_{45}\left(\frac{\partial w}{\partial y}+\theta_{y}\right)+k A_{55}\left(\frac{\partial w}{\partial x}+\theta_{x}\right)
\end{aligned}
$$

where $Q_{x}$ and $Q_{y}$ are the transverse shear forces, $M_{x}$ and $M_{y}$ are the bending moments, $M_{x y}$ is the twisting moment, $\bar{P}_{1 c d}$ is vertical reaction, $\bar{P}_{2 c d}, \bar{P}_{3 c d}$ are moment reactions around $x$-, $y$-axes, $k=5 / 6$ is the shear correction factor, $\delta\left(x-x_{q}\right)$ and $\delta\left(y-y_{r}\right)$ are Dirac's delta functions, $A_{i j}$ is the extensional stiffness $(i, j=4,5), D_{i j}$ is the bending stiffness $(i, j=1,2,6)$.

$A_{i j}, D_{i j}$ can be obtained by the following expressions.

$$
\begin{aligned}
& A_{i j}=Q_{i j} h, \quad D_{i j}=\frac{1}{12} Q_{i j} h^{3} \\
& \bar{Q}_{11}=Q_{11} c^{4}+2\left(Q_{12}+2 Q_{66}\right) c^{2} s^{2}+Q_{22} s^{4} \\
& \bar{Q}_{12}=Q_{12}\left(c^{4}+s^{4}\right)+\left(Q_{11}+Q_{22}-4 Q_{66}\right) c^{2} s^{2}, \\
& \bar{Q}_{16}=\left(Q_{11}-Q_{12}-2 Q_{66}\right) c^{3} s-\left(Q_{22}-Q_{12}-2 Q_{66}\right) c s^{3} \\
& \bar{Q}_{22}=Q_{11} s^{4}+2\left(Q_{12}+2 Q_{66}\right) c^{2} s^{2}+Q_{22} c^{4} \\
& \bar{Q}_{26}=\left(Q_{11}-Q_{12}-2 Q_{66}\right) c s^{3}-\left(Q_{22}-Q_{12}-2 Q_{66}\right) c^{3} s \\
& \bar{Q}_{66}=\left(Q_{11}+Q_{22}-2 Q_{12}-2 Q_{66}\right) c^{2} s^{2}+Q_{66}\left(c^{4}+s^{4}\right) \\
& \bar{Q}_{44}=Q_{44} c^{2}+Q_{55} s^{2}, \quad \bar{Q}_{45}=\left(Q_{55}-Q_{44}\right) c s \\
& \bar{Q}_{55}=Q_{44} s^{2}+Q_{55} c^{2}, \quad c=\cos \theta, \quad s=\sin \theta \\
& Q_{11}=\frac{E_{1}}{1-\nu_{12} \nu_{21}}, \quad Q_{22}=\frac{E_{2}}{1-\nu_{12} \nu_{21}}
\end{aligned}
$$




$$
\begin{aligned}
& Q_{12}=\frac{\nu_{12} E_{2}}{1-\nu_{12} \nu_{21}}, \quad Q_{44}=G_{23} \\
& Q_{55}=G_{31}, \quad Q_{66}=G_{12}
\end{aligned}
$$

where $E_{1}$ is the axial modulus in the 1-direction, $E_{2}$ is the axial modulus in the 2-direction, $\nu_{12}$ is the Poisson's ratio associated with loading in the 1-direction and strain in the 2-direction, $\nu_{21}$ is the Poisson's ratio associated with loading in the 2-direction and strain in the 1-direction, $G_{12}, G_{23}$ and $G_{31}$ are the shear moduli in 1-2, 2-3 and 1-3 planes.

By using the non-dimensional expressions

$$
\begin{aligned}
& {\left[X_{1}, X_{2}\right]=\frac{a^{2}}{D_{0}\left(1-\nu_{12} \nu_{21}\right)}\left[Q_{y}, Q_{x}\right]} \\
& {\left[X_{3}, X_{4}, X_{5}\right]=\frac{a}{D_{0}\left(1-\nu_{12} \nu_{21}\right)}\left[M_{x y}, M_{y}, M_{x}\right]} \\
& {\left[X_{6}, X_{7}, X_{8}\right]=\left[\theta_{y}, \theta_{x}, \frac{w}{a}\right], \quad[\eta, \zeta, \xi]=\left[\frac{x}{a}, \frac{y}{b}, \frac{z}{h}\right]}
\end{aligned}
$$

where $D_{0}=E_{2} h_{0}^{3} / 12\left(1-\nu_{12} \nu_{21}\right)$ is the standard bending rigidity, $h_{0}$ is the standard thickness of the plate.

The differential Eqs. (1) (8) can be rewritten as

$$
\begin{aligned}
\sum_{s=1}^{8} & \left\{F_{1 t s} \frac{\partial X_{s}}{\partial \zeta}+F_{2 t s} \frac{\partial X_{s}}{\partial \eta}+F_{3 t s} X_{s}\right\} \\
& +\sum_{f=1}^{3} \sum_{c=0}^{m} \sum_{d=0}^{n} P_{f c d} \delta\left(\eta-\eta_{c}\right) \delta\left(\zeta-\zeta_{d}\right) \delta_{f t} \\
& +P \delta\left(\eta-\eta_{q}\right) \delta\left(\zeta-\zeta_{r}\right) \delta_{1 t}=0 \quad(t=1 \sim 8)(9)
\end{aligned}
$$

where $\left[P, P_{1 c d}, P_{2 c d}, P_{3 c d}\right]=\left[\bar{P} a, \bar{P}_{1 c d} a, \bar{P}_{2 c d},-\bar{P}_{3 c d}\right]$ $/ D_{0}\left(1-\nu_{12} \nu_{21}\right) ; \delta_{f t}$ is Kronecker's delta, $F_{1 t s}, F_{2 t s}$ and $F_{3 t s}$ are given in Appendix A.

By dividing a rectangular plate vertically into $m$ equal-length parts and horizontally into $n$ equallength parts as shown in Fig. 2, the plate can be considered as a group of discrete points which are the intersections of the $(m+1)$-vertical and $(n+1)$ horizontal dividing lines. In this paper, the rectangular area, $0 \leq \eta \leq \eta_{i}, 0 \leq \zeta \leq \zeta_{j}$, corresponding to the arbitrary intersection $(i, j)$ as shown in Fig. 2 is denoted as the area $[i, j]$, the intersection $(i, j)$ denoted by $\bigcirc$ is called the main point of the area $[i, j]$, the intersections denoted by $\circ$ are called the inner dependent points of the area, and the intersections denoted by $\bullet$ are called the boundary dependent points of the area.

By integrating the equation (9) over the area $[i, j]$, the following integral equation is obtained.

$$
\begin{aligned}
\sum_{s=1}^{8} & \left\{F_{1 t s} \int_{0}^{\eta_{i}}\left[X_{s}\left(\eta, \zeta_{j}\right)-X_{s}(\eta, 0)\right] d \eta\right. \\
& +F_{2 t s} \int_{0}^{\zeta_{j}}\left[X_{s}\left(\eta_{i}, \zeta\right)-X_{s}(0, \zeta)\right] d \zeta
\end{aligned}
$$

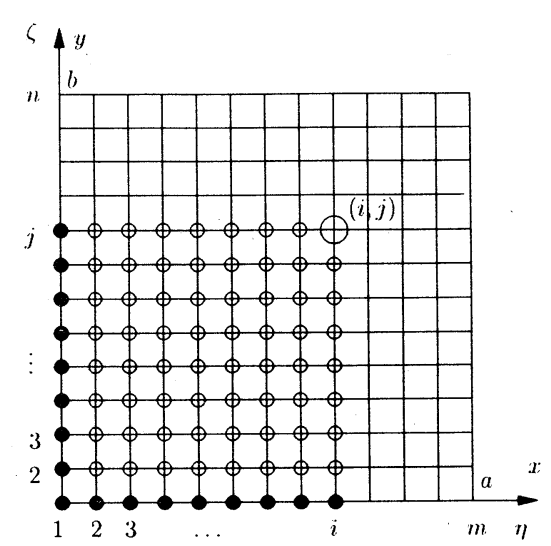

Fig. 2 Discrete points on a rectangular plate

$$
\begin{aligned}
& \left.+F_{3 t s} \int_{0}^{\eta_{i}} \int_{0}^{\zeta_{j}} X_{s}(\eta, \zeta) d \eta d \zeta\right\} \\
& +\sum_{f=1}^{3} \sum_{c=0}^{m} \sum_{d=0}^{n} P_{f c d} u\left(\eta-\eta_{c}\right) u\left(\zeta-\zeta_{d}\right) \delta_{f t} \\
& +P u\left(\eta-\eta_{q}\right) u\left(\zeta-\zeta_{r}\right) \delta_{1 t}=0
\end{aligned}
$$

where $u\left(\eta-\eta_{c}\right), u\left(\zeta-\zeta_{d}\right), u\left(\eta-\eta_{q}\right)$ and $u\left(\zeta-\zeta_{r}\right)$ are the unit step functions.

Next, by applying the numerical integration method, the simultaneous equation for the unknown quantities $X_{s i j}=X_{s}\left(\eta_{i}, \zeta_{j}\right)$ at the main point $(i, j)$ of the area $[i, j]$ is obtained as follows.

$$
\begin{aligned}
& \sum_{s=1}^{8}\left\{F_{1 t s} \sum_{k=0}^{i} \beta_{i k}\left(X_{s k j}-X_{s k 0}\right)\right. \\
& +F_{2 t s} \sum_{l=0}^{j} \beta_{j l}\left(X_{s i l}-X_{s 0 l}\right) \\
& \left.+F_{3 t s} \sum_{k=0}^{i} \sum_{l=0}^{j} \beta_{i k} \beta_{j l} X_{s k l}\right\} \\
& +\sum_{f=1}^{3} \sum_{c=0}^{m} \sum_{d=0}^{n} P_{f c d} u_{i c} u_{j d} \delta_{f t}+P u_{i q} u_{j r} \delta_{1 t}=0
\end{aligned}
$$

where $\beta_{i k}=\alpha_{i k} / m, \beta_{j l}=\alpha_{j l} / n, \alpha_{i k}=1-\left(\delta_{0 k}+\right.$ $\left.\delta_{i k}\right) / 2, \alpha_{j l}=1-\left(\delta_{0 l}+\delta_{j l}\right) / 2, t=1 \sim 8, i=1 \sim m$, $j=1 \sim n$.

The solution $X_{p i j}$ of the simultaneous equation (11) is

$$
\begin{aligned}
X_{p i j}= & \sum_{t=1}^{8}\left\{\sum_{k=0}^{i} \beta_{i k} A_{p t}\left[X_{t k 0}-X_{t k j}\left(1-\delta_{i k}\right)\right]\right. \\
& +\sum_{l=0}^{j} \beta_{j l} B_{p t}\left[X_{t 0 l}-X_{t i l}\left(1-\delta_{j l}\right)\right] \\
& \left.+\sum_{k=0}^{i} \sum_{l=0}^{j} \beta_{i k} \beta_{j l} C_{p t k l} X_{t k l}\left(1-\delta_{i k} \delta_{j l}\right)\right\}
\end{aligned}
$$




$$
-\sum_{f=1}^{3} \sum_{c=0}^{m} \sum_{d=0}^{n} \gamma_{p f} P_{f c d} u_{i c} u_{j d}-A_{p 1} P u_{i q} u_{j r}
$$

where $p=1 \sim 8, A_{p t}, B_{p t}, C_{p t k l}$ and $\gamma_{p f}$ are given in Appendix B.

In the equation (12), the quantity $X_{p i j}$ at the main point $(i, j)$ of the area $[i, j]$ is related to the quantities $X_{t k 0}$ and $X_{t 0 l}$ at the boundary dependent points of the area and the quantities $X_{t k j}, X_{t i l}$ and $X_{t k l}$ at the inner dependent points of the area. With the spreading of the area $[i, j]$ according to the regular order as $[1,1],[1,2], \cdots,[1, n],[2,1],[2,2], \cdots,[2, n]$, $\cdots,[m, 1],[m, 2], \cdots,[m, n]$, a main point of a smaller area becomes one of the inner dependent points of the following larger areas. Whenever the quantity $X_{p i j}$ at the main point $(i, j)$ is obtained by using the equation (12) in the above mentioned order, the quantities $X_{t k j}, X_{t i l}$ and $X_{t k l}$ at the inner dependent points of the following larger areas can be eliminated by substituting the obtained results into the corresponding terms of the right side of equation (12). By repeating this process, the equation $X_{p i j}$ at the main point is only related to the quantities $X_{r k 0}(r=1,3,4,6,7,8)$ and $X_{s 0 l}(s=2,3,5,6,7,8)$, which are six independent quantities at the each boundary dependent point along the horizontal axis and the vertical axis in Fig. 2, respectively. The result is

$$
\begin{aligned}
X_{p i j}= & \sum_{d=1}^{6}\left\{\sum_{f=0}^{i} a_{p i j f d} X_{r f 0}+\sum_{g=0}^{j} b_{p i j g d} X_{s 0 g}\right\} \\
& +\sum_{f=1}^{3} \sum_{c=0}^{m} \sum_{d=0}^{n} \bar{q}_{f p i j c d} P_{f c d}+\bar{q}_{p i j} P
\end{aligned}
$$

where $a_{p i j f d}, b_{p i j g d}, \bar{q}_{f p i j c d}$ and $\bar{q}_{p i j}$ are given in Appendix $\mathrm{C}$.

The equation (13) gives the discrete solution of the fundamental differential equation (9) of the bending problem of a plate under a concentrated load, and the discrete Green function is chosen as $X_{8 i j} /\left[\bar{P} a / D_{0}(1-\right.$ $\left.\nu_{12} \nu_{21}\right]$.

\section{Equivalent Rectangular Plate of a Triangular Plate}

An idea of equivalent rectangular plate is introduced to solve the bending problem of anisotropic right triangular plates. A right triangular plate is quite different from uniform rectangular plates, but it can be translated into equivalent rectangular plates with non-uniform thickness (shown in Fig. 3). In

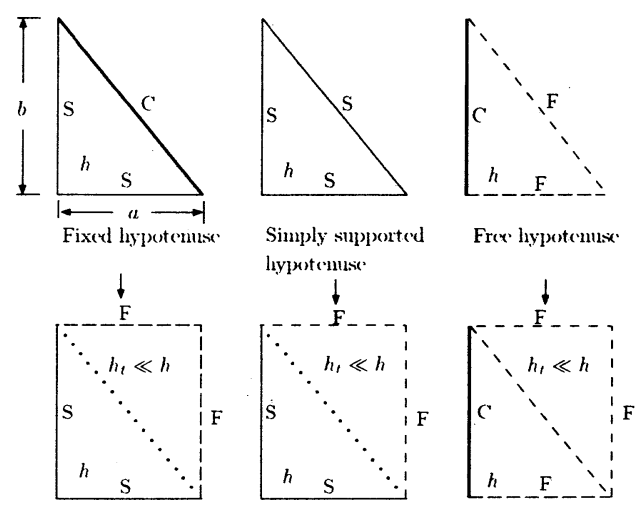

Fig. 3 Triangular plates and their equivalent rectangular plates (C: clamped edge; S: simply supported edge; F: free edge)

order to ensure half part of the equivalent rectangular plate has the same boundary and load conditions as the original triangular plate, some point supports shown by $(\cdots \cdots)$ at discrete points along the hypotenuse are used to enforce the boundary conditions along the original hypotenuse, the another half part of the equivalent rectangular plate with two free edges has very thin thickness and there is no load on it. The values of three reactions $P_{1 c d}, P_{2 c d}, P_{3 c d}$ at each point support can be determined by the following conditions.

$\theta_{t}=\theta_{n}=w=0, \quad$ if original hypotenuse is fixed.

$M_{n}=\theta_{t}=w=0, \quad$ if original hypotenuse is simply supported.

$P_{1 c d}=P_{2 c d}=P_{3 c d}=0$, if original hypotenuse is free.

The conditions $\theta_{t}=\theta_{n}=w=0$ mean that the slope around the tangential axis of the line of point supports, the slope around the normal axis of the line of point supports and the deflection at each point support are zero. The conditions $M_{n}=\theta_{t}=w=0$ mean that the bending moment around the tangential axis of the line of point supports, the slope around the normal axis of the line of point supports and the deflection at point support are zero. The conditions $P_{1 c d}=P_{2 c d}=P_{3 c d}=0$ mean that there is no point support along the hypotenuse.

The thickness of the actual part of original right triangular plate is expressed by $h$, the thickness of additional part of the equivalent rectangular plate is expressed as $h_{t}$ and the thickness at a point on the border line between the actual part and the additional part of the equivalent rectangular plate is taken as $\left(h+h_{t}\right) / 2$. The fixed, simply supported and free edges are denoted by the symbols $\mathrm{C}, \mathrm{S}, \mathrm{F}$, respectively and 
shown by thick solid line

solid line and dotted line - - . The first symbol indicates the conditions at $x=0$, the second at $y=0$ and the third at the hypotenuse.

\section{Characteristic Equation of Free Vi- bration of Rectangular Plate with Non-uniform Thickness}

By applying the Green function $w\left(x_{0}, y_{0}, x, y\right) / \bar{P}$ which is the displacement at a point $\left(x_{0}, y_{0}\right)$ of a plate with a concentrated load $\bar{P}$ at a point $(x, y)$ and point support at each discrete point $\left(x_{c}, y_{d}\right)$, the displacement amplitude $\hat{w}\left(x_{0}, y_{0}\right)$ at a point $\left(x_{0}, y_{0}\right)$ of the rectangular plate during the free vibration is given as $\hat{w}\left(x_{0}, y_{0}\right)=\int_{0}^{b} \int_{0}^{a} \rho h \omega^{2} \hat{w}(x, y)\left[w\left(x_{0}, y_{0}, x, y\right) / \bar{P}\right] d x d y$

where $\rho$ is the mass density of the plate material.

The non-dimensional expressions are used as,

$$
\begin{aligned}
& \lambda^{4}=\frac{\rho_{0} h_{0} \omega^{2} a^{4}}{D_{0}\left(1-\nu_{12} \nu_{21}\right)}, H(\eta, \zeta)=\frac{\rho(x, y)}{\rho_{0}} \frac{h(x, y)}{h_{0}} \\
& W(\eta, \zeta)=\frac{\hat{w}(x, y)}{a} \\
& G\left(\eta_{0}, \zeta_{0}, \eta, \zeta\right)=\frac{w\left(x_{0}, y_{0}, x, y\right)}{a} \frac{D_{0}\left(1-\nu_{12} \nu_{21}\right)}{\bar{P} a}
\end{aligned}
$$

here $\rho_{0}$ is the standard mass density.

By using the numerical integration method, equation (14) is discretely expressed as

$$
k W_{k l}=\sum_{i=0}^{m} \sum_{j=0}^{n} \beta_{m i} \beta_{n j} H_{i j} G_{k l i j} W_{i j}, \quad k=1 /\left(\mu \lambda^{4}\right)
$$

From equation (15) homogeneous linear equations in $(m+1) \times(n+1)$ unknowns $W_{00}, W_{01}, \cdots, W_{0 n}, W_{10}, W_{11}$ $\cdots, W_{1 n}, \cdots W_{m 0}, W_{m 1}, \cdots, W_{m n}$ are obtained as

$$
\begin{array}{r}
\sum_{i=0}^{m} \sum_{j=0}^{n}\left(\beta_{m i} \beta_{n j} H_{i j} G_{k l i j}-k \delta_{i k} \delta_{j l}\right) W_{i j}=0 \\
(k=0,1, \cdots, m, l=0,1, \cdots, n)
\end{array}
$$

The characteristic equation of the free vibration of a rectangular plate with variable thickness is obtained from the equation (16) as follows.

$$
\left(\begin{array}{ccccc}
\mathbf{K}_{00} & \mathbf{K}_{\mathbf{0 1}} & \mathbf{K}_{\mathbf{0 2}} & \ldots & \mathbf{K}_{\mathbf{0 m}} \\
\mathbf{K}_{10} & \mathbf{K}_{11} & \mathbf{K}_{12} & \ldots & \mathbf{K}_{\mathbf{1 m}} \\
\mathbf{K}_{\mathbf{2 0}} & \mathbf{K}_{\mathbf{2 1}} & \mathbf{K}_{\mathbf{2 2}} & \ldots & \mathbf{K}_{\mathbf{2 m}} \\
\vdots & \vdots & \vdots & \ddots & \vdots \\
\mathbf{K}_{\mathbf{m 0}} & \mathbf{K}_{\mathbf{m 1}} & \mathbf{K}_{\mathbf{m 2}} & \ldots & \mathbf{K}_{\mathbf{m m}}
\end{array}\right)=0
$$

where

$$
\mathbf{K}_{\mathrm{ij}}=\beta_{m j}\left[\begin{array}{ccc}
\beta_{n 0} H_{j 0} G_{i 0 j 0}-k \delta_{i j} & \beta_{n 1} H_{j 1} G_{i 0 j 1} \\
\beta_{n 0} H_{j 0} G_{i 1 j 0} & \beta_{n 1} H_{j 1} G_{i 1 j 1}-k \delta_{i j} \\
\beta_{n 0} H_{j 0} G_{i 2 j 0} & \beta_{n 1} H_{j 1} G_{i 2 j 1} \\
\vdots & \vdots \\
\beta_{n 0} H_{j 0} G_{i n j 0} & \beta_{n 1} H_{j 1} G_{i n j 1} \\
\beta_{n 2} H_{j 2} G_{i 0 j 2} & \cdots & \beta_{n n} H_{j n} G_{i 0 j n} \\
\beta_{n 2} H_{j 2} G_{i 1 j 2} & \cdots & \beta_{n n} H_{j n} G_{i 1 j n} \\
\beta_{n 2} H_{j 2} G_{i 2 j 2}-k \delta_{i j} & \cdots & \beta_{n n} H_{j n} G_{i 2 j n} \\
\vdots & \ddots & \vdots \\
\beta_{n 2} H_{j 2} G_{i n j 2} & \cdots & \beta_{n n} H_{j n} G_{i n j n}-k \delta_{i j}
\end{array}\right]
$$

\section{Numerical Results}

The results for the isotropic and anisotropic right triangular plates are given to show the convergence and accuracy of the numerical solution obtained by the present method. For the isotropic plate, the Poisson's ratio $\nu=0.3$. For the anisotropic plate, the graphite/epoxy material is used. Its properties are given as $E_{1} / E_{2}=17.57, G_{12} / E_{2}=G_{13} / E_{2}=$ $0.7, G_{23} / E_{2}=0.5, \nu_{12}=0.28$.

\subsection{Convergence of Solution}

In order to examine the convergence, numerical calculation is carried out by varying the number of divisions $m$ and $n$. The lowest eight natural frequency parameters of isotropic isosceles CFF plate are shown in Fig. 4. Fig. 5 is used to determine the suitable thickness ratio $h / h_{t}$ of the original and additional parts. Fig. 6 and Fig. 7 show the convergence for the orthotropic CFF plate. It can be noticed that convergent results can be obtained for CFF plate when $m$ and $n$ are not smaller than 10 and it is sufficient to set the thickness ratio $h / h_{t} \geq 5$. So, for CFF plate, the thickness ratio $h / h_{t}=5$ is used and the convergent values of frequency parameter are obtained by using Richardson's extrapolation formula for two cases of divisional numbers $m(=n)$ of 10 and 12 . By the same method, for SSC and SSS plates, the thickness ratio $h / h_{t}=5$ is also used but the convergent values of frequency parameter are obtained by using Richardson's extrapolation formula for two cases of divisional numbers $m(=n)$ of 14 and 16 .

\subsection{Accuracy of Solution}

To investigate the accuracy of the results obtained by the present method, frequencies and their mode shapes of isotropic and anisotropic triangular plates 


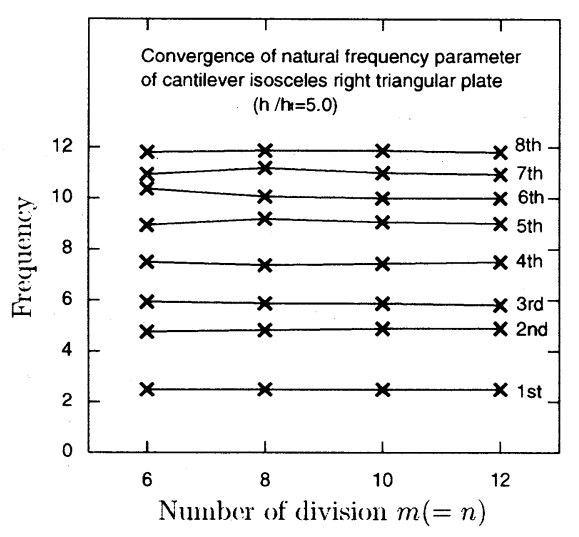

Fig. 4 Natural frequency parameter $\lambda$ versus the number of divison $m(=n)$ for isotropic $\mathrm{CFF}$ isosceles right triangular plate

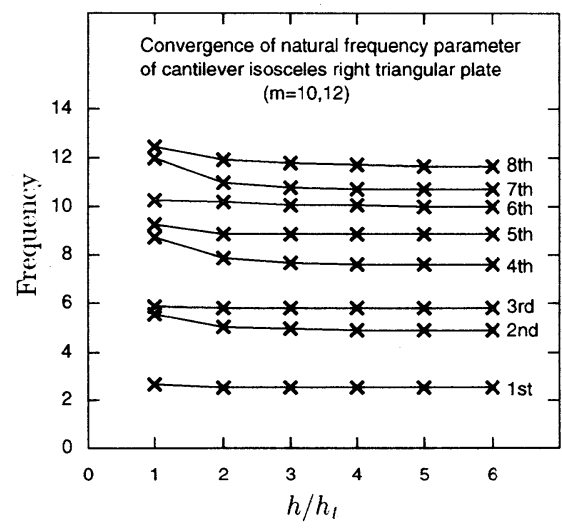

Fig. 5 Natural frequency parameter $\lambda$ versus the thickness ratio $h / h_{t}$ for isotropic CFF isosceles right triangular plate

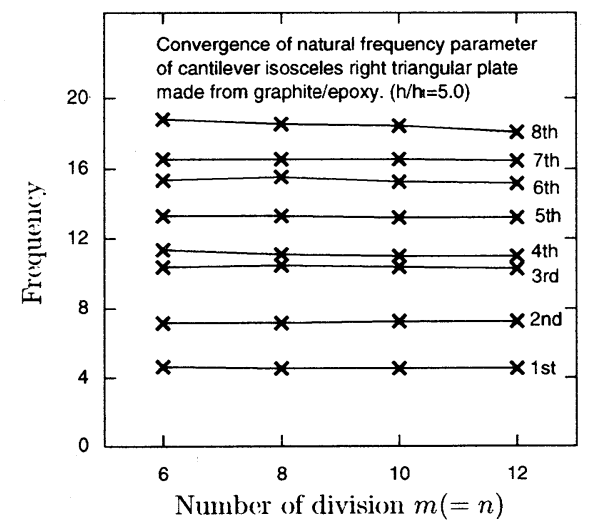

Fig. 6 Natural frequency parameter $\lambda$ versus the number of divison $m(=n)$ of orthotropic CFF isosceles right triangular plate

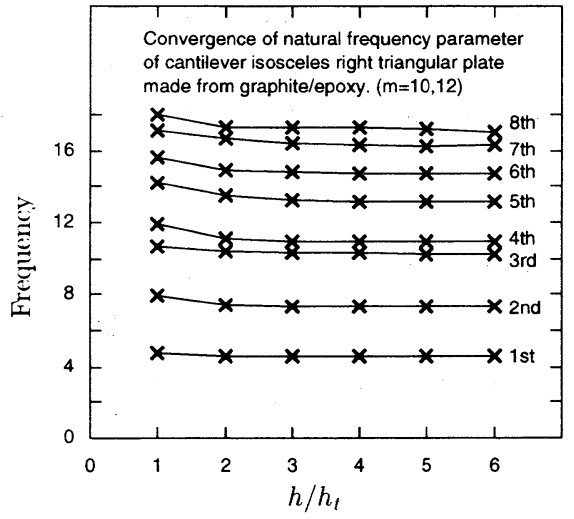

Fig. 7 Natural frequency $\lambda$ versus the thickness ratio $h / h_{t}$ for orthotropic CFF isosceles right triangular plate

Table 1 Natural frequency parameter $\lambda$ for isotropic $\mathrm{CFF}$ isosceles right triangular plate

\begin{tabular}{lccccc}
\hline & \multicolumn{5}{c}{ Mode sequence number } \\
\cline { 2 - 6 } References & 1 & 2 & 3 & 4 & 5 \\
\hline Present & & & & & \\
$10 \times 10$ & 2.498 & 4.860 & 5.850 & 7.466 & 9.059 \\
$12 \times 12$ & 2.507 & 4.875 & 5.833 & 7.502 & 8.997 \\
Ex. & 2.527 & 4.908 & 5.796 & 7.585 & 8.856 \\
Kim $^{7)}$ & 2.542 & 4.959 & 5.852 & 7.672 & 8.951 \\
Lam $^{11)}$ & 2.540 & 4.957 & 5.858 & 7.873 & - \\
\hline
\end{tabular}

Ex.: Richardson's extrapolation results.

Table 2 Natural frequency parameter $\lambda$ for isotropic SSC isosceles right triangular plate

\begin{tabular}{lccccc}
\hline & \multicolumn{5}{c}{ Mode sequence number } \\
\cline { 2 - 6 } References & 1 & 2 & 3 & 4 & 5 \\
\hline Present & & & & & \\
$14 \times 14$ & 8.667 & 12.132 & 13.541 & 16.112 & 17.143 \\
$16 \times 16$ & 8.590 & 11.924 & 13.355 & 15.661 & 16.727 \\
Ex. & 8.340 & 11.244 & 12.747 & 14.188 & 15.368 \\
Kim ${ }^{7)}$ & 8.305 & 11.267 & 12.726 & 14.378 & 15.575 \\
\hline
\end{tabular}

are presented. Plates with uniform and variable thickness are analyzed for two cases of thin and moderate thickness.

\section{(1) Right Triangular Plate with Uniform Thickness}

Table1 $\sim$ Table 4 give the numerical solutions for the lowest five frequencies of isotropic CFF, SSC, SSS and orthotropic CFF plates $(b / a=1)$. The thickness ratio $h / a$ is equal to 0.01 . The mode shapes of isotropic CFF, SSC and orthotropic CFF plates with 
Table 3 Natural frequency parameter $\lambda$ for isotropic SSS isosceles right triangular plate

\begin{tabular}{lccccc}
\hline & \multicolumn{5}{c}{ Mode sequence number } \\
\cline { 2 - 6 } References & 1 & 2 & 3 & 4 & 5 \\
\hline Present & & & & & \\
$14 \times 14$ & 7.430 & 10.757 & 12.221 & 14.534 & 15.628 \\
$16 \times 16$ & 7.384 & 10.620 & 12.086 & 14.213 & 15.325 \\
Ex. & 7.234 & 10.175 & 11.646 & 13.165 & 14.333 \\
Kim ${ }^{7)}$ & 7.193 & 10.174 & 11.608 & 13.314 & 14.490 \\
\hline
\end{tabular}

Table 4 Natural frequency parameter $\lambda$ for orthotropic $\mathrm{CFF}$ isosceles right triangular plate

\begin{tabular}{lccccc}
\hline & \multicolumn{5}{c}{ Mode sequence number } \\
\cline { 2 - 6 } References & 1 & 2 & 3 & 4 & 5 \\
\hline Present & & & & & \\
$10 \times 10$ & 4.631 & 7.236 & 10.355 & 10.075 & 13.266 \\
$12 \times 12$ & 4.630 & 7.250 & 10.331 & 11.042 & 13.227 \\
Ex. & 4.628 & 7.283 & 10.277 & 10.967 & 13.139 \\
Kim $^{7)}$ & 4.659 & 7.350 & 10.398 & 11.091 & 13.313 \\
\hline
\end{tabular}

Table 5 Natural frequency parameter $\lambda$ for isotropic CFF right triangular plate $(b / a=1.5)$

\begin{tabular}{lccccc}
\hline & \multicolumn{5}{c}{ Mode sequence number } \\
\cline { 2 - 6 } References & 1 & 2 & 3 & 4 & 5 \\
\hline Present & & & & & \\
$10 \times 10$ & 2.432 & 4.298 & 5.614 & 6.283 & 8.006 \\
$12 \times 12$ & 2.437 & 4.308 & 5.589 & 6.311 & 7.992 \\
Ex. & 2.447 & 4.333 & 5.532 & 6.374 & 7.959 \\
Kim $^{7)}$ & 2.464 & 4.372 & 5.588 & 6.449 & 8.072 \\
\hline
\end{tabular}

Table 6 Natural frequency parameter $\lambda$ for orthotropic CFF right triangular plate $(b / a=$ 1.5)

\begin{tabular}{lccccc}
\hline & \multicolumn{5}{c}{ Mode sequence number } \\
\cline { 2 - 6 } References & 1 & 2 & 3 & 4 & 5 \\
\hline Present & & & & & \\
$10 \times 10$ & 4.486 & 6.459 & 8.768 & 10.765 & 11.128 \\
$12 \times 12$ & 4.483 & 6.457 & 8.732 & 10.652 & 11.036 \\
Ex. & 4.476 & 6.454 & 8.652 & 10.396 & 10.826 \\
$\operatorname{Kim} 7)$ & 4.501 & 6.506 & 8.730 & 10.561 & 10.896 \\
\hline
\end{tabular}

aspect ratio $b / a=1$ are shown in Fig. 8. The mode shapes of SSS is the same as those of SSC. Table 5 and Table 6 give the natural frequency parameters of
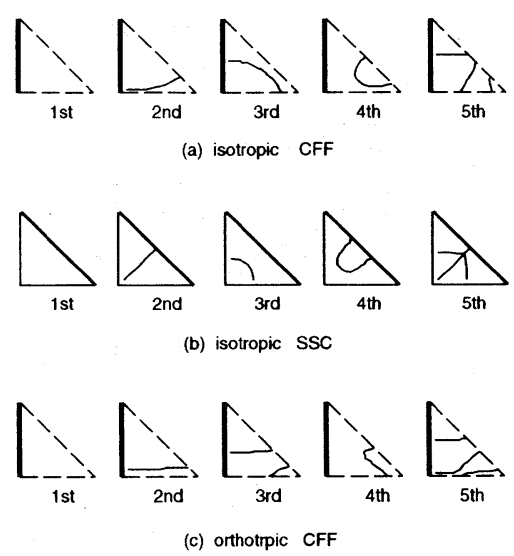

Fig. 8 Nodal patterns for isosceles right triangular plate
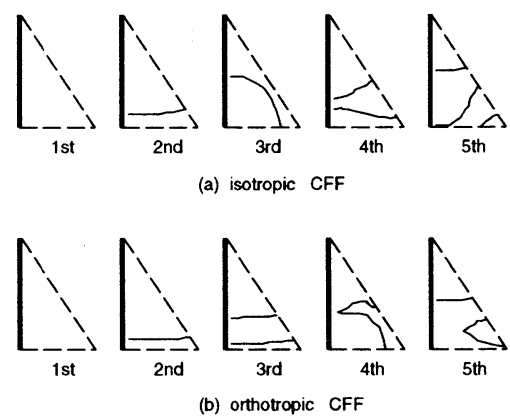

Fig. 9 Nodal patterns for CFF right triangular plate $(b / a=1.5)$

isotropic and orthotropic CFF plates with aspect ratio $b / a=1.5$, respectively. The mode shapes of them are shown in Fig. 9. The present results are compared with those obtained by Kim and Dickinson ${ }^{7)}$ and Lam, Liew and Chow ${ }^{11)}$. They are in close agreement.

Fig. $10 \sim$ Fig. 12 show that the variation of the lowest three frequencies of isosceles right CFF, SSC and SSS plates with fibre orientation. As the angle of fibre orientation $\theta$ increases from $0^{\circ}$ to $45^{\circ}$, the frequency decreases for CFF plate but increases for SSC and SSS plates. As $\theta$ increases from $45^{\circ}$ to $90^{\circ}$, the frequency decreases for all the plates. The first frequency reaches a maximum at $\theta=0^{\circ}$ for $\mathrm{CFF}$ plate and $\theta=45^{\circ}$ for SSC and SSS plates.

(2) Right Triangular Plate with Variable Thickness

In this paper, for the plate with variable thickness, its thickness varies linearly along the $x$-axis according to the equation $h(x, y)=h_{0}(1-\alpha x / a)$. 


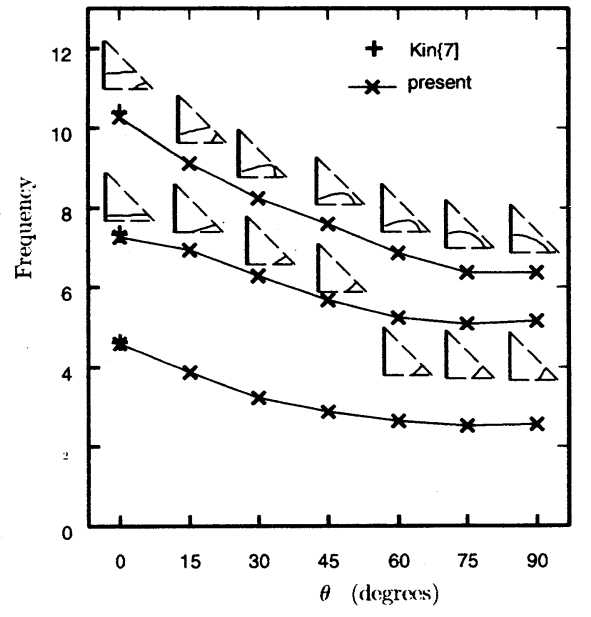

Fig. 10 The lowest three frequencies versus the fibre orientation for anisotropic $\mathrm{CFF}$ plate

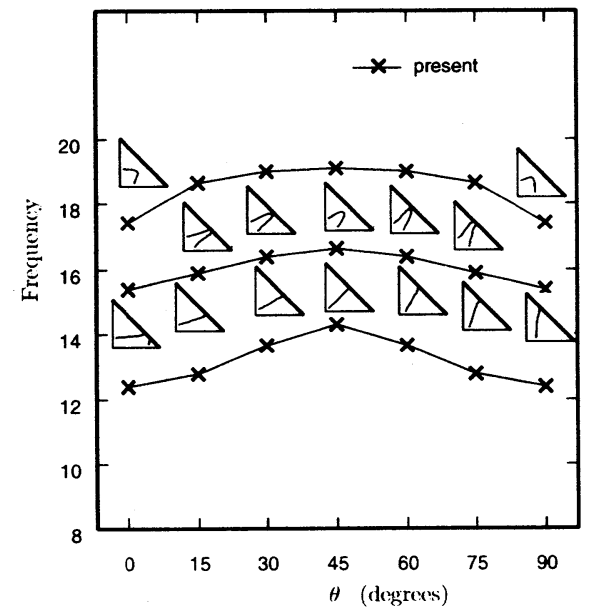

Fig. 11 The lowest three frequencies versus the fibre orientation for anisotropic SSC plate

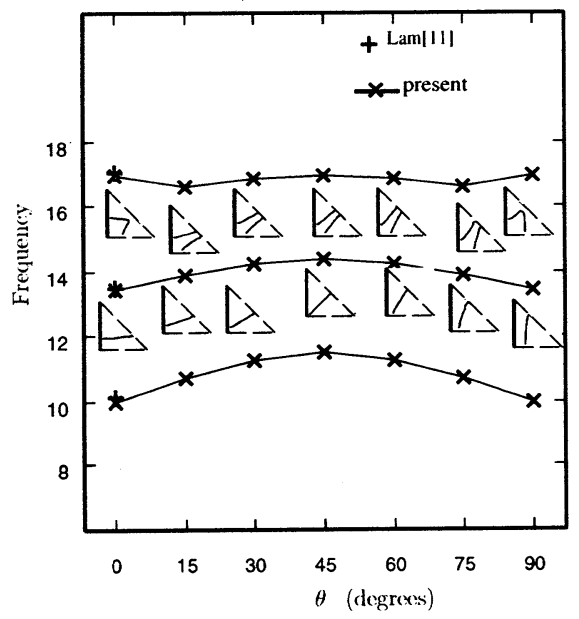

Fig. 12 The lowest three frequencies versus the fibre orientation for anisotropic SSS plate
Table 7 Natural frequency parameter $\lambda$ for isotropic CFF isosceles right triangular plate with variable thickness $(\alpha=0.5)$

\begin{tabular}{lccccc}
\hline & \multicolumn{5}{c}{ Mode sequence number } \\
\cline { 2 - 6 } References & 1 & 2 & 3 & 4 & 5 \\
\hline Present & & & & & \\
$10 \times 10$ & 2.471 & 4.520 & 5.340 & 6.812 & 8.098 \\
$12 \times 12$ & 2.486 & 4.529 & 5.339 & 6.816 & 8.023 \\
Ex. & 2.519 & 4.549 & 5.337 & 6.826 & 7.854 \\
Liew 9) & 2.582 & 4.636 & 5.479 & 6.906 & 8.002 \\
\hline
\end{tabular}

Table 8 Natural frequency parameter $\lambda$ for isotropic CFF isosceles right triangular plate with variable thickness $(\alpha=0.5, b / a=1.5)$

\begin{tabular}{lccccc}
\hline & \multicolumn{5}{c}{ Mode sequence number } \\
\cline { 2 - 6 } References & 1 & 2 & 3 & 4 & 5 \\
\hline Present & & & & & \\
$10 \times 10$ & 2.401 & 4.039 & 5.050 & 5.809 & 7.292 \\
$12 \times 12$ & 2.411 & 4.050 & 5.033 & 5.816 & 7.251 \\
Ex. & 2.436 & 4.076 & 4.996 & 5.832 & 7.157 \\
\hline
\end{tabular}

Table 9 Natural frequency parameter $\lambda$ for orthotropic CFF isosceles right triangular plate with variable thickness $(\alpha=0.5)$

\begin{tabular}{lccccc}
\hline & \multicolumn{5}{c}{ Mode sequence number } \\
\cline { 2 - 6 } References & 1 & 2 & 3 & 4 & 5 \\
\hline Present & & & & & \\
$10 \times 10$ & 4.575 & 6.873 & 9.520 & 10.008 & 12.164 \\
$12 \times 12$ & 4.588 & 6.891 & 9.472 & 9.994 & 12.091 \\
Ex. & 4.616 & 6.931 & 9.361 & 9.963 & 11.922 \\
\hline
\end{tabular}

Table 10 Natural frequency parameter $\lambda$ for orthotropic CFF right triangular plate with variable thickness $(\alpha=0.5, b / a=1.5)$

\begin{tabular}{lccccc}
\hline & \multicolumn{5}{c}{ Mode sequence number } \\
\cline { 2 - 6 } References & 1 & 2 & 3 & 4 & 5 \\
\hline Present & & & & & \\
$10 \times 10$ & 4.442 & 6.145 & 8.211 & 9.585 & 10.384 \\
$12 \times 12$ & 4.450 & 6.153 & 8.157 & 9.517 & 10.223 \\
Ex. & 4.470 & 6.173 & 8.036 & 9.361 & 9.859 \\
\hline
\end{tabular}

Table $7 \sim$ Table 10 give the natural frequency parameters of CFF right triangular plate with variable thickness $(\alpha=0.5)$ for aspect ratio $b / a=1$ and 1.5. The thickness ratio $h_{0} / a$ is equal to 0.01 . Isotropic 


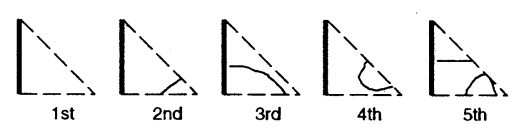

(a) $b / a=1$

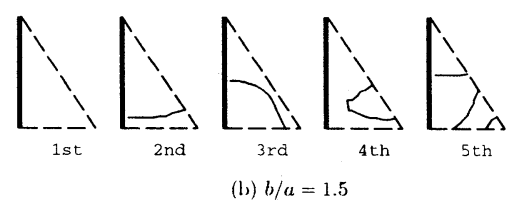

Fig. 13 Nodal patterns for isotropic CFF right triangular plate with variable thickness $(\alpha=$ $0.5)$

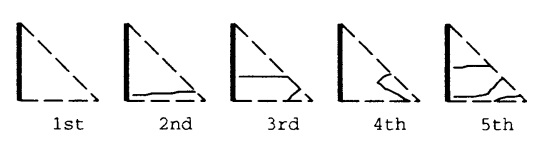

(a) $b / a=1$

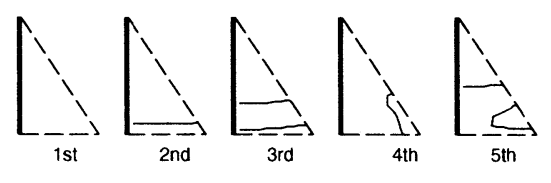

(b) $b / a=1.5$

Fig. 14 Nodal patterns for orthotropic CFF right triangular plate with variable thickness $(\alpha=$ $0.5)$

and orthotropic cases are considered. The results of isosceles right triangular plate for isotropic case are compared with those obtained by Liew, Lim and Lim ${ }^{9)}$ and it shows that they agree closely. The five mode shapes of these plates are shown in Fig. 13 and Fig. 14.

\section{(3) CFF Right Triangular Plate with Moder- ate Thickness}

Numerical solutions for the lowest five frequencies of isotropic and orthotropic CFF moderately thick plates with uniform and variable thickness $(\alpha=0.4)$ are shown in Table $11 \sim$ Table 14 . In this analysis, the aspect ratio $b / a=1$ and $h_{0} / a=0.006$ are used. By comparing the present results with those of McGee ${ }^{10)}$, it can be seen that a close agreement has been achieved. The mode shapes of these plates are similar to those of the plates with thin thickness.

\section{Conclusions}

An approximate method has been used for the free vibration of isotropic and orthotropic right triangular
Table 11 Natural frequency parameter $\lambda$ for isotropic CFF moderately thick triangular plate with uniform thickness

\begin{tabular}{lccccc}
\hline & \multicolumn{5}{c}{ Mode sequence number } \\
\cline { 2 - 6 } References & 1 & 2 & 3 & 4 & 5 \\
\hline Present & & & & & \\
$10 \times 10$ & 2.496 & 4.805 & 5.729 & 7.357 & 8.830 \\
$12 \times 12$ & 2.494 & 4.816 & 5.720 & 7.381 & 8.790 \\
Ex. & 2.487 & 4.841 & 5.700 & 7.433 & 8.697 \\
McGee ${ }^{10)}$ & 2.541 & 4.964 & 5.871 & 7.689 & 8.997 \\
\hline
\end{tabular}

Table 12 Natural frequency parameter $\lambda$ for isotropic CFF moderately thick triangular plate with variable thickness $(\alpha=0.4)$

\begin{tabular}{lccccc}
\hline & \multicolumn{5}{c}{ Mode sequence number } \\
\cline { 2 - 6 } References & 1 & 2 & 3 & 4 & 5 \\
\hline Present & & & & & \\
$10 \times 10$ & 2.477 & 4.562 & 5.364 & 6.879 & 8.165 \\
$12 \times 12$ & 2.487 & 4.575 & 5.353 & 6.904 & 8.137 \\
Ex. & 2.511 & 4.603 & 5.328 & 6.960 & 8.074 \\
McGee ${ }^{10)}$ & 2.569 & 4.713 & 5.580 & 7.095 & 8.242 \\
\hline
\end{tabular}

Table 13 Natural frequency parameter $\lambda$ for orthotropic $\mathrm{CFF}$ isosceles right triangular plate with uniform thickness

\begin{tabular}{lccccc}
\hline & \multicolumn{5}{c}{ Mode sequence number } \\
\cline { 2 - 6 } References & 1 & 2 & 3 & 4 & 5 \\
\hline Present & & & & & \\
$10 \times 10$ & 4.561 & 7.006 & 9.907 & 10.368 & 12.409 \\
$12 \times 12$ & 4.552 & 7.023 & 9.818 & 10.362 & 12.384 \\
Ex. & 4.537 & 7.052 & 9.661 & 10.351 & 12.338 \\
\hline
\end{tabular}

Table 14 Natural frequency parameter $\lambda$ for orthotropic $\mathrm{CFF}$ isosceles right triangular plate with variable thickness $(\alpha=0.4)$

\begin{tabular}{lccccc}
\hline & \multicolumn{5}{c}{ Mode sequence number } \\
\cline { 2 - 6 } References & 1 & 2 & 3 & 4 & 5 \\
\hline Present & & & & & \\
$10 \times 10$ & 4.524 & 6.769 & 9.418 & 9.785 & 11.863 \\
$12 \times 12$ & 4.532 & 6.788 & 9.291 & 9.776 & 11.733 \\
Ex. & 4.546 & 6.822 & 9.066 & 9.760 & 11.501 \\
\hline
\end{tabular}

plate with various aspect ratios and variable thickness. An equivalent rectangular plate is used to replace the right triangular plate. Point supports are introduced in the fundamental differential equations and used to enforce the boundary condition along the 
hypotenuse. Green function, which is the solution for the deflection, is used to obtain the characteristic equation of free vibration. Some frequency parameters and their mode shapes are shown in Tables and Figures. It can be seen the boundary condition, aspect ratio, the variation of thickness and the angle of the fibre orientation have great effects on the frequency parameters. Boundary condition also affects the trend of the variation of the frequency with fibre orientation. For the plates with different boundary conditions, the angle of the fibre orientation at which the first frequency reaches a maximum may be quite different. The results by the present method have been compared with those previously reported. It shows that the present results have a good convergence and satisfactory accuracy.

\section{Acknowledgements}

The present study is sponsored by the Japan Society for the Promotion of Science (JSPS).

\section{REFERENCES}

1) LEISSA 1969 Vibration of plates (NASA SP-160). Washington, D.C.: Office of Technology Utilization, NASA.

2) D.J.Gorman 1983 Journal of Sound and Vibration 89, 107-118. A highly accurate analytical solution for free vibration analysis of simply supported right triangular plates.

3) D.J.Gorman 1986 Journal of Sound and Vibration 106, 419-431. Free vibration analysis of right triangular plates with combinations of clampedsimply supported boundary conditions.

4) D.J.Gorman 1987 Journal of Sound and Vibration 112, 173-176. A modified superposition method for the free vibration analysis of right triangular plates.

5) H.T.SALIBA 1990 Journal of Sound and Vibration 139, 289-297. Transverse free vibration of simply supported right triangular thin plates: a highly accurate simplified solution.

6) H.T.SAliba 1995 Journal of Sound and Vibration 183, 765-778. Transverse free vibration of right triangular thin plates with combinations of clamped and simply supported boundary conditions: a highly accurate simplified solution.

7) C.S.KIM and S.M.DiCkinson 1990 Journal of Sound and Vibration 141, 291-311. The free flexural vibration of right triangular isotropic and or- thotropic plates.

8) S.MirzA and M.BiJlani 1985 Computers and Structures 21, 1129-1135. Vibration of triangular plates of variable thickness.

9) K.M.LiEw, C.W.LiM and M.K.Lim 1994 Journal of Sound and Vibration 177, 479-501. Transverse vibration of trapezoidal plates of variable thickness: unsymmetric trapezoids.

10) O.G.MCGEE and G.T.Giaimo 1992 Journal of Sound and Vibration 159, 279-293. Threedimensional vibrations of cantilevered right triangular plates.

11) K.Y.LAM, K.M.LIEW and S.T.CHOW 1990 Int.J.Mech.Sci. 32, 455-464. Free vibrations analysis of isotropic and orthotropic triangular plates.

12) S.K.Malhotra, N.Ganesan and M.A.Veluswami 1989 Composite Structures 12, 17-25. Vibrations of orthotropic triangular plates.

13) D.V.Bambill, P.A.A.Lauraand R.E.Rossi 1998 Journal of Sound and Vibration 210, 286-290. Transverse vibrations of rectangular, trapezoidal and triangular orthotropic, cantilever plates.

14) T.SAKIYAMA and M.HuANG 1998 Journal of Sound and Vibration 216, 379-397. Free vibration analysis of rectangular plates with variable thickness.

15) T.SAKIYAMA and M.HuAng 2000 Journal of Sound and Vibration 234, 841-858. Free vibration analysis of right triangular plates with variable thickness.

\section{Appendix A}

$$
\begin{aligned}
& F_{111}=F_{123}=F_{134}=1 \\
& F_{146}=\bar{D}_{12}, F_{147}=\bar{D}_{16} \\
& F_{156}=\bar{D}_{22}, F_{157}=F_{166}=\bar{D}_{26}, \\
& F_{167}=\bar{D}_{66}, F_{178}=k \bar{A}_{44} F_{188}=k \bar{A}_{45} \\
& F_{212}=F_{225}=F_{233}=\mu, F_{246}=F_{267}=\mu \bar{D}_{16} \\
& F_{247}=\mu \bar{D}_{11} F_{256}=\mu \bar{D}_{26}, F_{257}=\mu, \bar{D}_{12}, \\
& F_{266}=\mu \bar{D}_{66} F_{278}=F_{30907}=F_{31006}=\mu k \bar{A}_{45}, \\
& F_{288}=F_{387}=\mu k \bar{A}_{55}, \\
& F_{322}=F_{331}=-\mu, F_{345}=F_{354}=F_{363}=-\mu \bar{D} \\
& F_{371}=F_{382}=-\mu \overline{D T} \\
& \text { other } F_{k t s}=0
\end{aligned}
$$




\section{Appendix B}

$$
\begin{aligned}
& A_{p 1}=\gamma_{p 1}, A_{p 2}=0, A_{p 3}=\gamma_{p 2}, \\
& A_{p 4}=\gamma_{p 3}, A_{p 5}=0 \text {, } \\
& A_{p 6}=\bar{D}_{12} \gamma_{p 4}+\bar{D}_{22} \gamma_{p 5}+\bar{D}_{26} \gamma_{p 6} \text {, } \\
& A_{p 7}=\bar{D}_{16} \gamma_{p 06}+\bar{D}_{26} \gamma_{p 07}+\bar{D}_{66} \gamma_{p 08} \text {, } \\
& A_{p 8}=k\left(\bar{A}_{44} \gamma_{p 7}+\bar{A}_{45} \gamma_{p 8}\right) \\
& B_{p 1}=0, B_{p 2}=\mu \gamma_{p 1}, B_{p 3}=\mu \gamma_{p 3} \text {, } \\
& B_{p 4}=0, B_{p 5}=\mu \gamma_{p 2} \text {, } \\
& B_{p 6}=\mu\left(\bar{D}_{16} \gamma_{p 4}+\bar{D}_{26} \gamma_{p 5}+\bar{D}_{66} \gamma_{p 6}\right) \text {, } \\
& B_{p 7}=\mu\left(\bar{D}_{11} \gamma_{p 4}+\bar{D}_{12} \gamma_{p 5}+\bar{D}_{16} \gamma_{p 6}\right) \text {, } \\
& B_{p 8}=\mu k\left(\bar{A}_{45} \gamma_{p 7}+\bar{A}_{55} \gamma_{p 8}\right) \text {, } \\
& C_{p 1 k l}=\mu \gamma_{p 3}+\mu \overline{D T}_{k l} \gamma_{p 7} \text {, } \\
& C_{p 2 k l}=\mu \gamma_{p 2}+\mu \overline{D T}_{k l} \gamma_{p 8}, \\
& C_{p 3 k l}=\mu \bar{D}_{k l} \gamma_{p 6} \text {, } \\
& C_{p 4 k l}=\mu \bar{D}_{k l} \gamma_{p 7}, \\
& C_{p 5 k l}=\mu \bar{D}_{k l} \gamma_{p 4} \\
& C_{p 6 k l}=-\mu k\left(\bar{A}_{44} \gamma_{p 7}+\bar{A}_{45} \gamma_{p 8}\right. \text {, } \\
& C_{p 7 k l}=-\mu k\left(\bar{A}_{45} \gamma_{p 7}+\bar{A}_{55} \gamma_{p 8}\right) \text {, } \\
& C_{p 8 k l}=0\left[\gamma_{p t}\right]=\left[\rho_{t p}\right]^{-1} \text {, } \\
& \rho_{11}=\beta_{i i}, \rho_{12}=-\mu \beta_{j j}, \rho_{22}=-\mu \beta_{i j} \text {, } \\
& \rho_{23}=\beta_{i i}, \rho_{25}=\mu \beta_{j j}, \rho_{31}=-\mu \beta_{i j} \text {, } \\
& \rho_{33}=\mu \beta_{j j}, \rho_{34}=\beta_{i i}, \rho_{45}=-\mu \beta_{i j} \bar{D}_{i j} \text {, } \\
& \rho_{46}=\bar{D}_{12} \beta_{i i}+\mu \bar{D}_{16} \beta_{j j}, \rho_{47}=\bar{D}_{16} \beta_{i i}+\mu \bar{D}_{11} \beta_{j j} \text {, } \\
& \rho_{54}=-\mu \beta_{i j} \bar{D}_{i j}, \rho_{56}=\bar{D}_{22} \beta_{i i}+\mu \bar{D}_{26} \beta_{j j} \text {, } \\
& \rho_{57}=\bar{D}_{26} \beta_{i i}+\mu \bar{D}_{12} \beta_{j j}, \rho_{63}=-\mu \beta_{i j} \bar{D}_{i j} \text {, } \\
& \rho_{666}=\bar{D}_{26} \beta_{i i}+\mu \bar{D}_{66} \beta_{j j}, \rho_{67}=\bar{D}_{66} \beta_{i i}+\mu \bar{D}_{16} \beta_{j j} \text {, } \\
& \rho_{71}=-\mu \beta_{i j} \bar{D}_{i j}, \rho_{76}=\mu k \bar{A}_{44} \beta_{i j} \text {, } \\
& \rho_{77}=\mu k \bar{A}_{45} \beta_{i j}, \rho_{78}=k\left(\bar{A}_{44} \beta_{i i}+\mu \bar{A}_{45} \beta_{j j}\right), \\
& \rho_{82}=-\mu \beta_{i j} \bar{D}_{i j}, \rho_{86}=\mu k \bar{A}_{45} \beta_{i j} \text {, } \\
& \rho_{87}=\mu k \bar{A}_{55} \beta_{i j}, \rho_{88}=k\left(\bar{A}_{45} \beta_{i i}+\mu \bar{A}_{55} \beta_{j j}\right), \\
& \text { other } \rho_{t p}=0
\end{aligned}
$$

\section{Appendix C}

$$
\begin{aligned}
& a_{1 i 0 i 01}= a_{3 i 0 i 02}=a_{4 i 0 i 03}=1 \\
& a_{6 i 0 i 04}= a_{7 i 0 i 05}=a_{8 i 0 i 06}=1, \\
& b_{20 j j 01}= b_{30 j j 02}=b_{50 j j 03}=1 \\
& b_{60 j j 04}= b_{70 j j 05}=b_{80 j j 06}=1, \\
& b_{300002}= 0 \\
& a_{p i j f d}= \sum_{t=1}^{13}\left\{\sum_{k=0}^{i} \beta_{i k} A_{p t}\left[a_{t k 0 f d}-a_{t k j f d}\left(1-\delta_{k i}\right)\right]\right. \\
& \quad+\sum_{l=0}^{j} \beta_{j l} B_{p t}\left[a_{t 0 l f d}-a_{t i l f d}\left(1-\delta_{l j}\right)\right] \\
&\left.\quad+\sum_{k=0}^{i} \sum_{l=0}^{j} \beta_{i k} \beta_{j l} C_{p t k l} a_{t k l f d}\left(1-\delta_{k i} \delta_{l j}\right)\right\} \\
& b_{p i j f d}=\sum_{t=1}^{13}\left\{\sum_{k=0}^{i} \beta_{i k} A_{p t}\left[b_{t k 0 g d}-b_{t k j g d}\left(1-\delta_{k i}\right)\right]\right.
\end{aligned}
$$

$$
\begin{aligned}
& +\sum_{l=0}^{j} \beta_{j l} B_{p t}\left[b_{t 0 l g d}-b_{t i l g d}\left(1-\delta_{l j}\right)\right] \\
& \left.+\sum_{k=0}^{i} \sum_{l=0}^{j} \beta_{i k} \beta_{j l} C_{p t k l} b_{t k l g d}\left(1-\delta_{k i} \delta_{l j}\right)\right\}
\end{aligned}
$$$$
\bar{q}_{f p i j c d}=\sum_{e=1}^{8}\left\{\sum_{k=0}^{i} \beta_{i k} A_{p e}\left[\bar{q}_{f e k 0 c d}-\bar{q}_{f e k j c d}\left(1-\delta_{k i}\right)\right]\right.
$$$$
+\sum_{l=0}^{j} \beta_{j l} B_{p e}\left[\bar{q}_{f e 0 l c d}-\bar{q}_{f e i l c d}\left(1-\delta_{l j}\right)\right]
$$$$
\left.+\sum_{k=0}^{i} \sum_{l=0}^{j} \beta_{i k} \beta_{j l} C_{p e k l} \bar{q}_{f e k l c d}\left(1-\delta_{k i} \delta_{l j}\right)\right\}
$$$$
-\gamma_{p f} u_{i k} u_{j r} \bar{u}_{f k l}
$$

$$
\begin{aligned}
\bar{q}_{p i j}=\sum_{t=1}^{13}\left\{\sum_{k=0}^{i} \beta_{i k} A_{p t}\left[\bar{q}_{t k 0}-\bar{q}_{t k j}\left(1-\delta_{k i}\right)\right]\right. \\
+\sum_{l=0}^{j} \beta_{j l} B_{p t}\left[\bar{q}_{t 0 l}-\bar{q}_{t i l}\left(1-\delta_{l j}\right)\right] \\
+\sum_{k=0}^{i} \sum_{l=0}^{j} \beta_{i k} \beta_{j l} C_{p t k l}-A_{p 1} u_{i q} u_{j r}
\end{aligned}
$$

(Received April 20,2001) 